\title{
From hypotonic maintenance fluid to severe hyponatremia: a case report
}

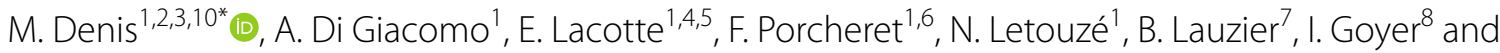 \\ D. Brossier ${ }^{1,9}$
}

\begin{abstract}
Background: The principles for maintenance intravenous fluid prescription in children were developed in the 1950s. These guidelines based on the use of hypotonic solutions have been challenged regularly for they seem to be associated with an increased risk of hospital-acquired hyponatremia.

Case presentation: We report the case of a 4-week-old Caucasian child admitted for acute bronchiolitis who received hypotonic maintenance fluids and developed severe hyponatremia $(94 \mathrm{mmol} / \mathrm{L})$ with hyponatremic encephalopathy.
\end{abstract}

Conclusion: This clinical situation can serve as a reminder of the latest recommendations from the American Academy of Pediatrics regarding the use of intravenous fluids that promote the use of isotonic fluids in children.

Keywords: Hyponatremia, Hypotonic fluid, Intensive care, Isotonic fluid

\section{Background}

Maintenance intravenous fluids (MIVF) prescription practices are highly variable among pediatricians [1]. For decades, MIVF prescriptions were based on the 1957 Holliday and Segar recommendations [2]. These guidelines recommended the use of hypotonic fluids as a standard of practice for MIVF in children. Since 1957, this dogma has been constantly challenged for it appears to be associated with severe episodes of hospital-acquired hyponatremia [3, 4]. Besides hyponatremia, wrongful usage of intravenous fluid therapy can be associated with pericardial effusion and ascites, which can also be caused or enhanced by malignancy and sepsis [57]. Severe hyponatremia is defined as a plasma sodium level $<125 \mathrm{mmol} / \mathrm{L}$ and is associated with hyponatremic encephalopathy $[8,9]$, a spectrum of symptoms related to cerebral edema. Severe hyponatremia is a medical

\footnotetext{
*Correspondence: manon.denis@chu-nantes.fr

${ }^{10}$ Service de reanimation pédiatrique, CHU de Nantes, 4ème étage bâtiment HME, 38 boulevard Jean-Monnet, 44093 Nantes Cedex 1, France

Full list of author information is available at the end of the article
}

emergency that can lead to irreversible brain injury or death, if not managed promptly [8]. To help avoid this critical iatrogenic complication, the American Academy of Pediatrics published a guideline regarding MIVF in 2018, making only one and very simple recommendation: prescribe isotonic intravenous fluids in children [10]. Despite its straightforwardness, this simple recommendation remains neglected. We present the case of a 4-week-old child admitted for acute bronchiolitis who received hypotonic MIVF and then developed hyponatremic encephalopathy.

\section{Case presentation}

A 4-week-old, $2.8 \mathrm{~kg}$ Caucasian girl presented to the emergency department of a general hospital with a 1-day history of upper airway infection and poor feeding. She was born at 35 weeks of gestational age with a birth weight of $2.380 \mathrm{~kg}$, Apgar 10, in a context of emergency C-section subsequent to preeclampsia and had no risk factor for mother-to-infant infection. She was admitted to the pediatric ward with a diagnosis of mild bronchiolitis with signs of rhinitis and reduced original author(s) and the source, provide a link to the Creative Commons licence, and indicate if changes were made. The images or other third party material in this article are included in the article's Creative Commons licence, unless indicated otherwise in a credit line to the material. If material is not included in the article's Creative Commons licence and your intended use is not permitted by statutory regulation or exceeds the permitted use, you will need to obtain permission directly from the copyright holder. To view a copy of this licence, visit http://creativecommons.org/licenses/by/4.0/. The Creative Commons Public Domain Dedication waiver (http://creativeco mmons.org/publicdomain/zero/1.0/) applies to the data made available in this article, unless otherwise stated in a credit line to the data. 
food intake, but showed no sign of dehydration. Upon admission, the patient was apyretic $\left(37.5{ }^{\circ} \mathrm{C}\right)$, heart rate (HR) was at 172 beats per minute, blood pressure (BP) was $90 / 50 \mathrm{mmHg}$, and respiratory rate (RR) was 32 breaths/minute. She had no signs of hypoperfusion, and heart sounds were regular, with no audible murmur.

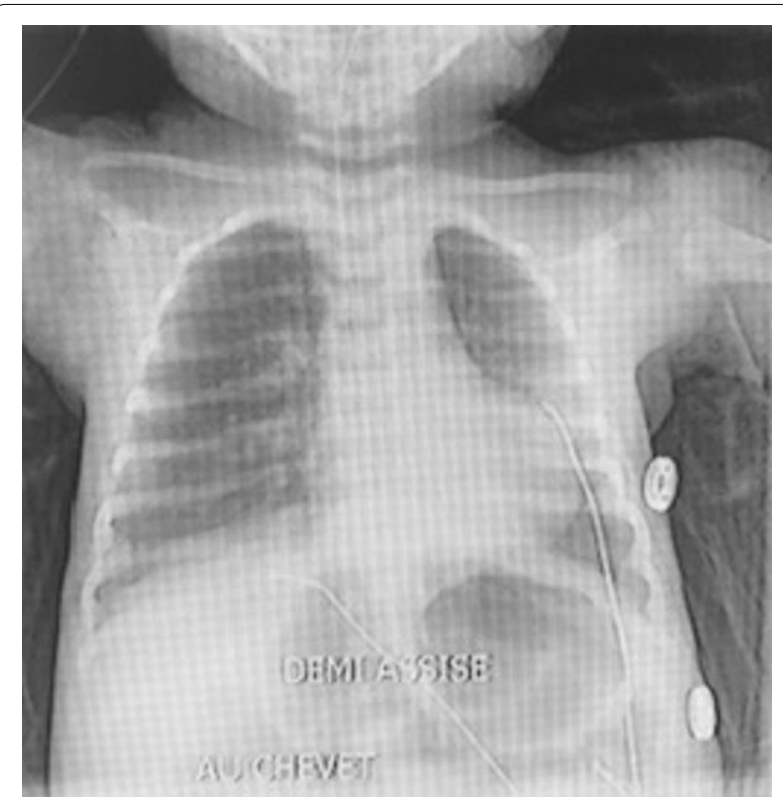

Fig. 1 Chest $X$-ray upon admission to the pediatric ward
Respiratory examination showed bilateral crackles, with moderate respiratory distress signs, and saturation was $100 \%$ under oxygen therapy $0.5 \mathrm{~L} /$ minute. She had moderate axial hypotonia, normal cries, and normotensive anterior fontanelle. There were no other findings on physical examination. Chest X-ray showed a chest distension without apparent infectious site (Fig. 1). Respiratory panel test was negative, including for respiratory syncytial virus (RSV) and influenza, as well as aerobic and anaerobic blood cultures. Blood tests at admission reported hyponatremia and hyperkalemia $\left(\mathrm{Na}^{+}\right.$ $126 \mathrm{mmol} / \mathrm{L}, \mathrm{K}^{+} 5.6 \mathrm{mmol} / \mathrm{L}$, bicarbonate $22.3 \mathrm{mmol} / \mathrm{L}$, creatinine $20 \mu \mathrm{mol} / \mathrm{L}$, urea $5.4 \mathrm{mmol} / \mathrm{L}$, white blood cells (WBC) $9.2 \times 10^{9} / \mathrm{L}$, hemoglobin $(\mathrm{Hb}) 10.9 \mathrm{~g} / \mathrm{dL}$, platelets (Plt) $553 \times 10^{9} / \mathrm{L}, \mathrm{C}$-reactive protein $(\mathrm{CRP})<5 \mathrm{mg} / \mathrm{L}$ ) (Fig. 2). She received continuous intravenous infusion of hypotonic fluid (sodium chloride $34 \mathrm{mmol} / \mathrm{L}$, potassium $20 \mathrm{mmol} / \mathrm{L}$, calcium $2.26 \mathrm{mmol} / \mathrm{L}$, and glucose $5 \%$ ) at a rate of $122 \mathrm{~mL} / \mathrm{kg} /$ day, plus enteral feeding via nasogastric tube $(130 \mathrm{~mL} / \mathrm{kg} /$ day $)$, supplemental oxygen $0.5 \mathrm{~L} /$ minute, respiratory physiotherapy, and oral betamethasone ( $0.375 \mathrm{mg}$ once daily for 2 days).

After 48 hours, she was found drowsy and hypotonic, still reactive to stimulation with no other sign of intracranial hypertension. She presented respiratory distress, intermittent desaturation, hypothermia $\left(35.5^{\circ} \mathrm{C}\right)$, and normal hemodynamic parameters (HR 140 beats per minute and BP 95/50 $\mathrm{mmHg}$ ). The blood tests showed: glycemia $6.10 \mathrm{mmol} / \mathrm{L}, \mathrm{Na}^{+} 94 \mathrm{mmol} / \mathrm{L}, \mathrm{K}^{+} 5.2 \mathrm{mmol} / \mathrm{L}$,

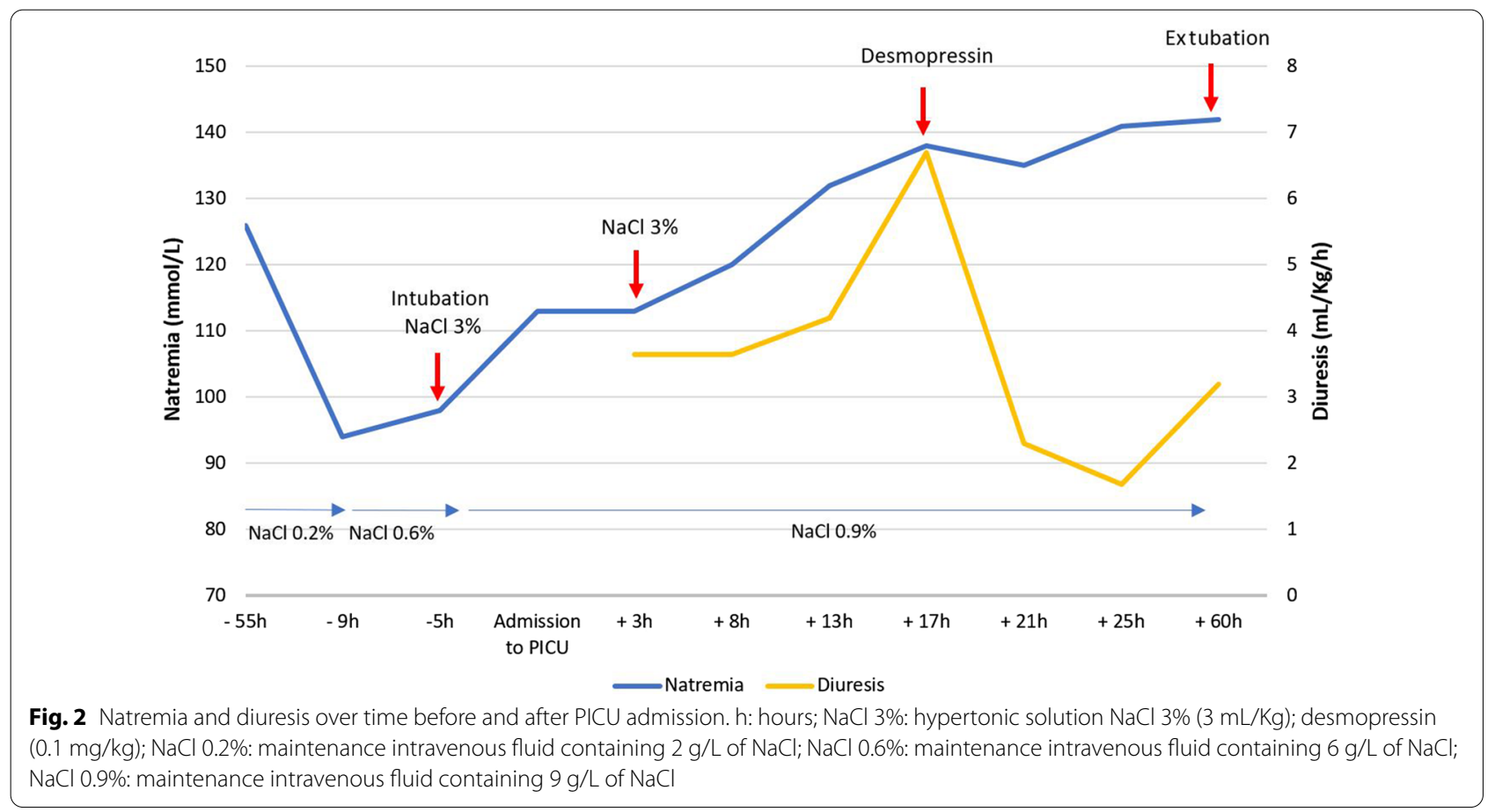


$\mathrm{Cl}^{-} 67 \mathrm{mmol} / \mathrm{L}$, osmolality $194.1 \mathrm{mmol} / \mathrm{kg}$, creatinine $10 \mu \mathrm{mol} / \mathrm{L}$, urea $3 \mathrm{mmol} / \mathrm{L}$, WBC $18.4 \times 10^{9} / \mathrm{L}, \mathrm{Hb}$ $10.8 \mathrm{~g} / \mathrm{dL}$, Plt $734 \times 10^{9} / \mathrm{L}, \mathrm{CRP}<5 \mathrm{mg} / \mathrm{L}$ and urine tests: $\mathrm{Na}^{+} 48 \mathrm{mmol} / \mathrm{L}, \mathrm{K}^{+} 50 \mathrm{mmol} / \mathrm{L}, \mathrm{Cl}^{-} 102 \mathrm{mmol} / \mathrm{L}$ and osmolarity $456 \mathrm{mosm} / \mathrm{L}$. $\mathrm{NaCl}$ was added to the MIVF (total $\mathrm{Na}^{+}$content: $100 \mathrm{mmol} / \mathrm{L}$ ), total intravenous intakes were restricted to $85 \mathrm{~mL} / \mathrm{kg} /$ day, and feedings were stopped. Cerebral tomodensitometry showed widespread cerebral edema (Fig. 3). Four hours later, the blood tests showed persistent severe hyponatremia $(98 \mathrm{mmol} / \mathrm{L})$. Patient transfer to the Pediatric Intensive Care Unit (PICU) was then decided. Upon arrival of the pediatric transport team, the child was unconscious (Glasgow Coma Scale: 8) and presented respiratory pauses and hemodynamic symptoms of intracranial hypertension (HR $115 \mathrm{bpm}, \mathrm{BP}$ 110/60 mmHg). Auscultation revealed a decrease of the left vesicular murmur, and hemodynamics remained stable. No other abnormal clinical signs were found. She immediately received osmotherapy ( $3 \mathrm{~mL} / \mathrm{kg}$ of $\mathrm{NaCl} 3 \% \mathrm{w} / \mathrm{v})$. She was then sedated with propofol $(3 \mathrm{mg} / \mathrm{kg})$ and paralyzed with succinylcholine $(1 \mathrm{mg} / \mathrm{kg})$ for rapid sequence intubation and intubated with a 3.5-cuffed tracheal tube. Afterwards, she received isotonic MIVF (dextrose 5\% with $\mathrm{NaCl} 145 \mathrm{mmol} / \mathrm{L}$ ) with a total intake restricted to $80 \mathrm{~mL} / \mathrm{kg} /$ day. Upon PICU admission, she was sedated with continuous infusions of midazolam $(30 \mu \mathrm{g} / \mathrm{kg} /$ hour) and morphine (30 $\mu \mathrm{g} / \mathrm{kg} /$ hour $)$, without any clinical sign of intracranial hypertension.
Three hours after admission, transcranial Doppler ultrasound (TDU) showed elements of intracranial hypertension (pulsatility index (PI): 1.40, diastolic velocity $\left(V_{\mathrm{d}}\right): 16 \mathrm{~cm} /$ second). TDU was normalized (PI: 1.02, $V_{\mathrm{d}}: 30 \mathrm{~cm} /$ second) after a second IV bolus of $\mathrm{NaCl} 3 \%$ $\mathrm{w} / \mathrm{v}(3 \mathrm{~mL} / \mathrm{kg})$.

Thirteen hours postadmission, the patient presented with transient polyuria $(5.4 \mathrm{~mL} / \mathrm{kg} /$ hour$)$ associated with a decrease in urinary density to $1.005 \mathrm{~g} / \mathrm{mL}$, urinary $\mathrm{Na}^{+}<10 \mathrm{mmol} / \mathrm{L}$, and a rapid increase in natremia (Fig. 2). Intravenous desmopressin $0.1 \mu \mathrm{g} / \mathrm{kg}$ was given to control polyuria and stabilize natremia. Subsequently, diuresis and natremia normalized with isotonic MIVF and fluid restriction for 2 days until resumption of enteral feedings.

Amplitude-integrated electroencephalography (aEEG) did not report any comitial activity and sedation was weaned 24 hours after admission. The infant showed signs of awakening. She was extubated 2 days after admission, and then received supplemental oxygen for 5 days and systemic steroids (intravenous dexamethasone $0.15 \mathrm{mg} / \mathrm{kg}$ every 6 hours for 24 hours) for postextubation laryngeal stridor.

Three days after admission, the child's EEG reported elements of cerebral suffering and some left central acute elements for which epileptic origin was not confirmed. EEG performed on day 6 was normal. Magnetic resonance imaging (MRI), performed on day 4 , showed significant regression of cerebral vasogenic edema with multiple small areas of supratentorial cytotoxic edema,

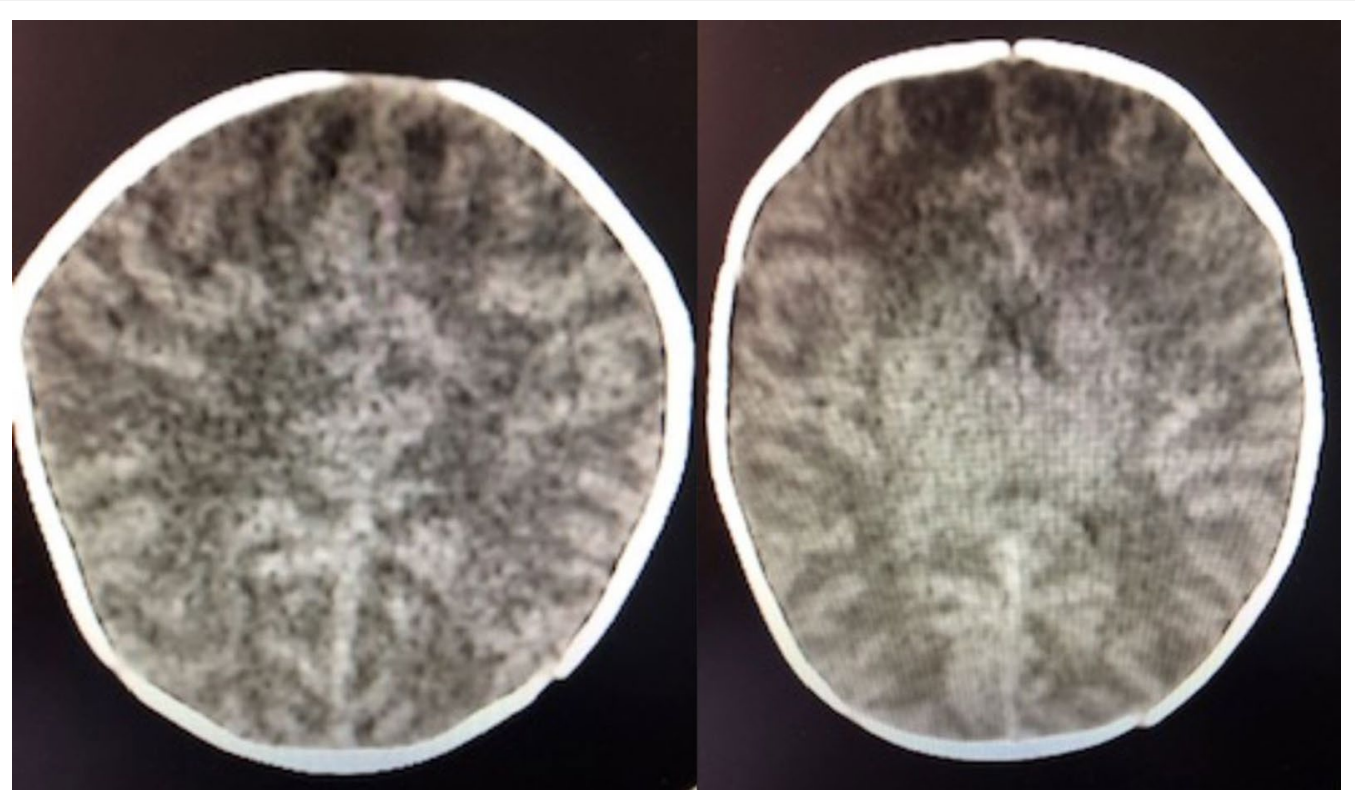

Fig. 3 Cerebral tomodensitometry depicting widespread cerebral edema 
whose distribution did not match any typical aspect of osmotic demyelination syndrome (ODS) and were imputed to ischemic injuries. This lack of ODS-related lesion was later confirmed by another MRI 6 months later.

SIAD assessment was performed with elimination of dysthyroidism [TSH $3.16 \mathrm{mUI} / \mathrm{L}(\mathrm{N})$ ] and adrenal insufficiency [8 a.m. cortisol: $52.3 \mu \mathrm{g} / \mathrm{L}(\mathrm{N})$ ], but antidiuretic hormone dosage was not available. Blood samples remained normal afterwards.

Eight days after PICU admission, neurological examination was normal. The infant was transferred to the general pediatric ward on day 8 and discharged home on day 12. In the weeks following discharge, she presented with feeding difficulties and suffered episodes of acute discomfort with desaturation, bradycardia, and laryngeal spasm requiring rehospitalization in the pediatric ward and then in the PICU. Upon admission, HR was at $175 \mathrm{bpm}$, BP 120/68 mmHg, RR 40/minute, and saturation 100\% under high-flow oxygen therapy with $\mathrm{FiO}_{2} 30 \%$. She had inspiratory dyspnea with severe respiratory distress, and other clinical signs were normal. Faced with a serious acute airway obstruction episode and an inability to intubate, urgent tracheostomy was performed. A subglottic stenosis was later found (Fig. 4). A tracheostomy remained in place until the age of 14 months. She had multiple dilations of the subglottic stenosis with good clinical examination. She suffers from moderate asthma and is fed by a gastrostomy because of an orality disorder.

\section{Discussion}

Isotonic maintenance intravenous fluid is recommended for hydration of hospitalized children [10]. We report here the case of a 4-week-old child who received hypotonic intravenous fluid and experienced secondary hyponatremia as well as dramatic neurological and respiratory complications, which led to a 1-year hospitalization period. This case demonstrates that following the guidelines regarding the use of isotonic fluid therapy is mandatory.

Over the past 60 years, infants and children received hypotonic MIVF as a standard of care, according to outdated recommendations that were based on theoretical calculations that were never validated in clinical trials. Even though numerous studies since the late 1990s have confirmed that hypotonic MIVF contributes to hospitalacquired hyponatremia [3, 4], these solutions are still commonly used in children owing to a fear of hypernatremia, hyperchloremic acidosis, or fluid overload [1, $11,12]$. The use of hypotonic intravenous solutions is all the more inappropriate since hospitalized children commonly present with SIAD [13] and reports of death or permanent neurological impairment arising from iatrogenic hyponatremia in both children and adults are
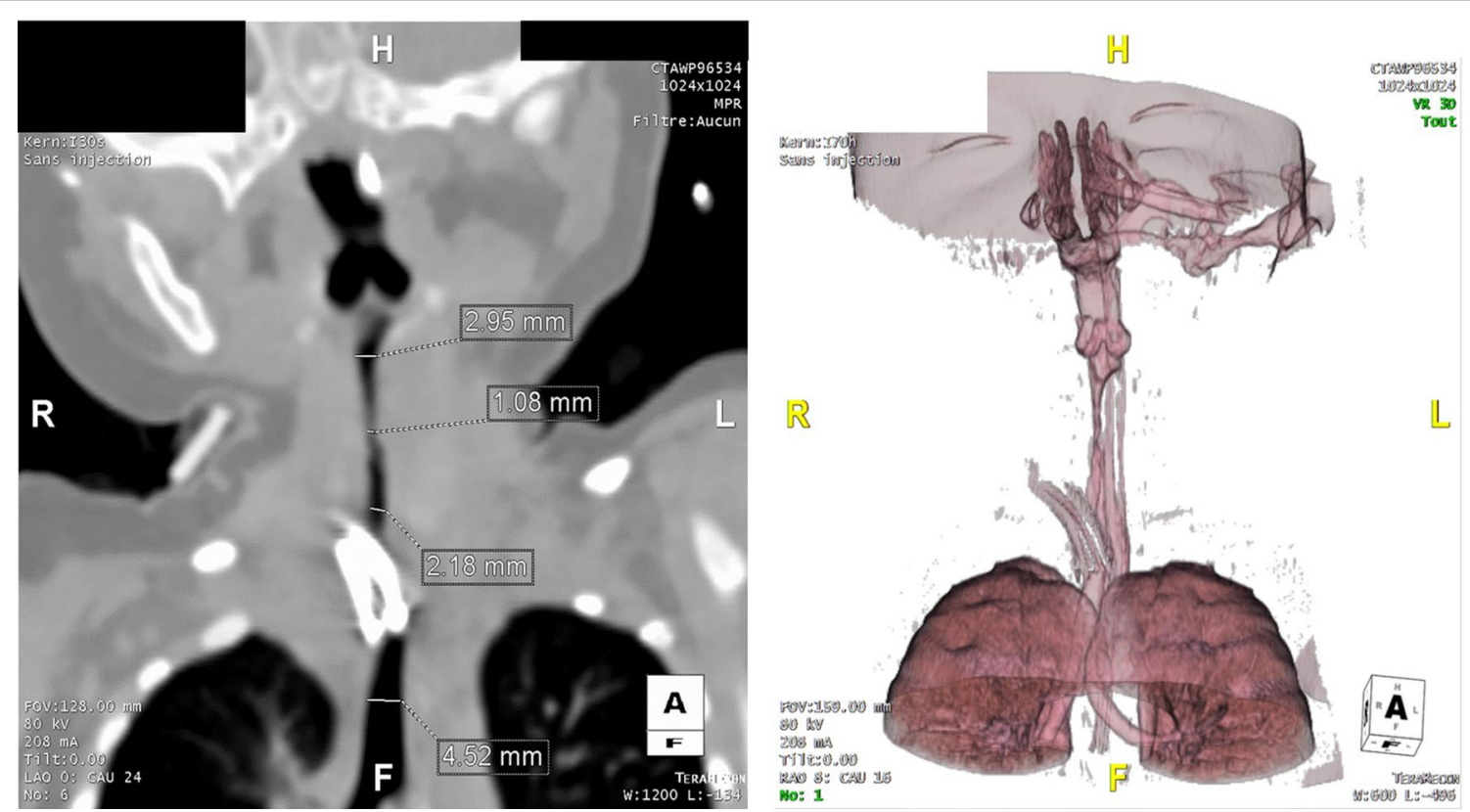

Fig. 4 Tomodensitometry and three-dimensional (3D) reconstruction of the subglottic stenosis

results. At 2 years of age, she has a normal neurological

documented [14]. The National Patient Safety Agency in 
Table 1 Available maintenance intravenous fluid solution and necessary additives

\begin{tabular}{|c|c|c|c|c|c|}
\hline Solution & Composition & $\begin{array}{l}\text { Osmolarity } \\
\text { (mOsmol/L) }\end{array}$ & $\begin{array}{l}\text { Necessary additives for } \\
\text { use in children (glucose } \\
4-5 \%+\text { near isotonic) }\end{array}$ & $\begin{array}{l}\text { Estimated final } \\
\text { composition }\end{array}$ & $\begin{array}{l}\text { Estimated final } \\
\text { osmolarity } \\
\text { (mOsmol/L) }\end{array}$ \\
\hline Human plasma & $\begin{array}{l}\text { Glucose } 3.5-6.1 \mathrm{mmol} / \mathrm{L} \\
\mathrm{Na}^{+} 135-144 \mathrm{mmol} / \mathrm{L} \\
\mathrm{Cl}^{-} 95-105 \mathrm{mmol} / \mathrm{L} \\
\mathrm{K}^{+} 3.5-5.3 \mathrm{mmol} / \mathrm{L} \\
\text { Bicarbonate } 23-30 \mathrm{mmol} / \mathrm{L} \\
\mathrm{Ca}^{2+} 2.2 \mathrm{mmol} / \mathrm{L} \\
\mathrm{Mg}^{2+} 0.8-1.2 \mathrm{mmol} / \mathrm{L}\end{array}$ & 308 & & & \\
\hline Normal saline $e^{a}$ & $\begin{array}{l}\mathrm{Na}^{+} 154 \mathrm{mmol} / \mathrm{L} \\
\mathrm{Cl}^{-} 154 \mathrm{mmol} / \mathrm{L}\end{array}$ & 308 & $\begin{array}{l}80 \mathrm{~mL} \text { of glucose } 30 \% \text { per } \\
500 \mathrm{~mL}\end{array}$ & $\begin{array}{l}\text { Glucose } 4.1 \% \\
\mathrm{Na}^{+} 133 \mathrm{mmol} / \mathrm{L} \\
\mathrm{Cl}^{-} 133 \mathrm{mmol} / \mathrm{L}\end{array}$ & 499 \\
\hline Normal saline G5\% ${ }^{a}$ & $\begin{array}{l}\text { Glucose } 5 \% \\
\mathrm{Na}^{+} 154 \mathrm{mmol} / \mathrm{L} \\
\mathrm{Cl}^{-} 154 \mathrm{mmol} / \mathrm{L}\end{array}$ & 580 & $\begin{array}{l}\text { Nothing to add, but unbal- } \\
\text { anced in anions, and does } \\
\text { not contain potassium or } \\
\text { calcium }\end{array}$ & & \\
\hline Ringer's lactate ${ }^{M D, a, b}$ & $\begin{array}{l}\mathrm{Na}^{+} 130 \mathrm{mmol} / \mathrm{L} \\
\mathrm{K}^{+} 4 \mathrm{mmol} / \mathrm{L} \\
\mathrm{Ca}^{2+} 1.4 \mathrm{mmol} / \mathrm{L} \\
\mathrm{Cl}^{-} 108 \mathrm{mmol} / \mathrm{L} \\
\text { Lactate } 27 \mathrm{mmol} / \mathrm{L}\end{array}$ & 253 & $\begin{array}{l}80 \mathrm{~mL} \text { of glucose } 30 \% \text { per } \\
500 \mathrm{~mL}\end{array}$ & $\begin{array}{l}\text { Glucose } 4.1 \% \\
\mathrm{Na}^{+} 112 \mathrm{mmol} / \mathrm{L} \\
\mathrm{K}^{+} 3.5 \mathrm{mmol} / \mathrm{L} \\
\mathrm{Ca}^{2+} 1.2 \mathrm{mmol} / \mathrm{L} \\
\mathrm{Cl}^{-} 93 \mathrm{mmol} / \mathrm{L} \\
\text { Lactate } 23 \mathrm{mmol} / \mathrm{L}\end{array}$ & 452 \\
\hline Ringer's lactate $\mathrm{G} 5 \%{ }^{\mathrm{MD}, \mathrm{a}, \mathrm{b}}$ & $\begin{array}{l}\text { Glucose } 5 \% \\
\mathrm{Na}^{+} 130 \mathrm{mmol} / \mathrm{L} \\
\mathrm{K}^{+} 4 \mathrm{mmol} / \mathrm{L} \\
\mathrm{Ca}^{2+} 1.5 \mathrm{mmol} / \mathrm{L} \\
\mathrm{Cl}^{-} 109 \mathrm{mmol} / \mathrm{L} \\
\text { Lactate } 28 \mathrm{mmol} / \mathrm{L}\end{array}$ & 525 & $\begin{array}{l}\text { Nothing to add, but slightly } \\
\text { hypotonic }\end{array}$ & & \\
\hline Hartmann's solution MD,a,b & $\begin{array}{l}\mathrm{Na}^{+} 131 \mathrm{mmol} / \mathrm{L} \\
\mathrm{K}^{+} 5.4 \mathrm{mmol} / \mathrm{L} \\
\mathrm{Ca}^{2+} 1.8 \mathrm{mmol} / \mathrm{L} \\
\mathrm{Cl}^{-} 111.7 \mathrm{mmol} / \mathrm{L} \\
\text { Lactate } 27.8 \mathrm{mmol} / \mathrm{L}\end{array}$ & 278 & $\begin{array}{l}80 \mathrm{~mL} \text { of glucose } 30 \% \text { per } \\
500 \mathrm{~mL}\end{array}$ & $\begin{array}{l}\text { Glucose } 4.1 \% \\
\mathrm{Na}^{+} 113 \mathrm{mmol} / \mathrm{L} \\
\mathrm{K}^{+} 4.7 \mathrm{mmol} / \mathrm{L} \\
\mathrm{Ca}^{2+} 1.6 \mathrm{mmol} / \mathrm{L} \\
\mathrm{Cl}^{-} 96.3 \mathrm{mmol} / \mathrm{L} \\
\text { Lactate } 24 \mathrm{mmol} / \mathrm{L}\end{array}$ & 466 \\
\hline Ringer's acetate $\mathrm{MD}, \mathrm{a}, \mathrm{b}^{-}$ & $\begin{array}{l}\mathrm{Na}^{+} 137 \mathrm{mmol} / \mathrm{L} \\
\mathrm{K}^{+} 4 \mathrm{mmol} / \mathrm{L} \\
\mathrm{Ca}^{2+} 1.65 \mathrm{mmol} / \mathrm{L} \\
\mathrm{Mg}^{2+} 1.25 \mathrm{mmol} / \mathrm{L} \\
\mathrm{Cl}^{-} 110 \mathrm{mmol} / \mathrm{L} \\
\text { Acetate } 36.8 \mathrm{mmol} / \mathrm{L}\end{array}$ & 291 & $\begin{array}{l}80 \mathrm{~mL} \text { of glucose } 30 \% \text { per } \\
500 \mathrm{~mL}\end{array}$ & $\begin{array}{l}\text { Glucose } 4.1 \% \\
\mathrm{Na}^{+} 118 \mathrm{mmol} / \mathrm{L} \\
\mathrm{K}^{+} 3.5 \mathrm{mmol} / \mathrm{L} \\
\mathrm{Ca}^{2+} 1.4 \mathrm{mmol} / \mathrm{L} \\
\mathrm{Mg}^{2+} 1.1 \mathrm{mmol} / \mathrm{L} \\
\mathrm{Cl}^{-} 94.8 \mathrm{mmol} / \mathrm{L} \\
\text { Acetate } 31.7 \mathrm{mmol} / \mathrm{L}\end{array}$ & 475 \\
\hline ELOMEL Isoton MD,a,b & $\begin{array}{l}\mathrm{Na}^{+} 140 \mathrm{mmol} / \mathrm{L} \\
\mathrm{K}^{+} 5 \mathrm{mmol} / \mathrm{L} \\
\mathrm{Mg}^{2+} 1.5 \mathrm{mmol} / \mathrm{L} \\
\mathrm{Ca}^{2+} 2.5 \mathrm{mmol} / \mathrm{L} \\
\mathrm{Cl}^{-} 108 \mathrm{mmol} / \mathrm{L} \\
\text { Acetate } 45 \mathrm{mmol} / \mathrm{L}\end{array}$ & 302 & $\begin{array}{l}80 \mathrm{~mL} \text { of glucose } 30 \% \text { per } \\
500 \mathrm{~mL}\end{array}$ & $\begin{array}{l}\text { Glucose } 4.1 \% \\
\mathrm{Na}^{+} 121 \mathrm{mmol} / \mathrm{L} \\
\mathrm{K}^{+} 4.3 \mathrm{mmol} / \mathrm{L} \\
\mathrm{Mg}^{2+} 1.3 \mathrm{mmol} / \mathrm{L} \\
\mathrm{Ca}^{2+} 2.2 \mathrm{mmol} / \mathrm{L} \\
\mathrm{Cl}^{-} 93.1 \mathrm{mmol} / \mathrm{L} \\
\text { Acetate } 38.8 \mathrm{mmol} / \mathrm{L}\end{array}$ & 485 \\
\hline
\end{tabular}


Table 1 (continued)

\begin{tabular}{|c|c|c|c|c|c|}
\hline Solution & Composition & $\begin{array}{l}\text { Osmolarity } \\
\text { (mOsmol/L) }\end{array}$ & $\begin{array}{l}\text { Necessary additives for } \\
\text { use in children (glucose } \\
4-5 \%+\text { near isotonic) }\end{array}$ & $\begin{array}{l}\text { Estimated final } \\
\text { composition }\end{array}$ & $\begin{array}{l}\text { Estimated final } \\
\text { osmolarity } \\
\text { (mOsmol/L) }\end{array}$ \\
\hline \multirow{7}{*}{$\begin{array}{l}\text { Plasma-Lyte } 148^{\text {MD,a,b }} \\
\text { Normosol }^{M D, a, b} \\
\text { Isolyte }^{\mathrm{MD}, a, b}\end{array}$} & $\mathrm{Na}^{+} 140 \mathrm{mmol} / \mathrm{L}$ & \multirow[t]{7}{*}{294} & \multirow{7}{*}{$\begin{array}{l}80 \mathrm{~mL} \text { of glucose } 30 \% \text { per } \\
500 \mathrm{~mL}\end{array}$} & Glucose $4.1 \%$ & \multirow[t]{14}{*}{479} \\
\hline & $\mathrm{K}^{+} 5 \mathrm{mmol} / \mathrm{L}$ & & & $\mathrm{Na}^{+} 121 \mathrm{mmol} / \mathrm{L}$ & \\
\hline & $\mathrm{Mg}^{2+} 1.5 \mathrm{mmol} / \mathrm{L}$ & & & $\mathrm{K}^{+} 4.3 \mathrm{mmol} / \mathrm{L}$ & \\
\hline & $\mathrm{Cl}^{-} 98 \mathrm{mmol} / \mathrm{L}$ & & & $\mathrm{Mg}^{2+} 1.3 \mathrm{mmol} / \mathrm{L}$ & \\
\hline & Acetate $27 \mathrm{mmol} / \mathrm{L}$ & & & $\mathrm{Cl}^{-} 84.5 \mathrm{mmol} / \mathrm{L}$ & \\
\hline & \multirow[t]{2}{*}{ Gluconate 23 mmol/L } & & & Acetate $23.3 \mathrm{mmol} / \mathrm{L}$ & \\
\hline & & & & Gluconate $19.8 \mathrm{mmol} / \mathrm{L}$ & \\
\hline \multirow[t]{7}{*}{ Plasmalyte 148 G5\% MD,a,b } & Glucose 5\% & \multirow[t]{7}{*}{572} & \multirow{7}{*}{$\begin{array}{l}\text { Nothing to add, but dose } \\
\text { not contain calcium }\end{array}$} & & \\
\hline & $\mathrm{Na}^{+} 140 \mathrm{mmol} / \mathrm{L}$ & & & & \\
\hline & $\mathrm{K}^{+} 5 \mathrm{mmol} / \mathrm{L}$ & & & & \\
\hline & $\mathrm{Mg}^{2+} 1.5 \mathrm{mmol} / \mathrm{L}$ & & & & \\
\hline & $\mathrm{Cl}^{-} 98 \mathrm{mmol} / \mathrm{L}$ & & & & \\
\hline & Acetate 27 mmol/L & & & & \\
\hline & Gluconate $23 \mathrm{mmol} / \mathrm{L}$ & & & & \\
\hline \multirow{8}{*}{$\begin{array}{l}\text { Isofundine } \mathrm{MD}^{\mathrm{MD}, \mathrm{a}} \mathrm{b} \\
\text { Ringerfundine } \mathrm{M}^{\mathrm{MD}, \mathrm{a}, \mathrm{b}} \\
\text { Sterofundine } \mathrm{MD}^{\mathrm{M}, \mathrm{a}, \mathrm{b}}\end{array}$} & $\mathrm{Na}^{+} 145 \mathrm{mmol} / \mathrm{L}$ & \multirow[t]{8}{*}{309} & \multirow[t]{8}{*}{$\begin{array}{l}80 \mathrm{~mL} \text { of glucose } 30 \% \text { per } \\
500 \mathrm{~mL}\end{array}$} & Glucose $4.1 \%$ & \multirow[t]{8}{*}{499} \\
\hline & $\mathrm{K}^{+} 4 \mathrm{mmol} / \mathrm{L}$ & & & $\mathrm{Na}^{+} 125 \mathrm{mmol} / \mathrm{L}$ & \\
\hline & $\mathrm{Ca}^{2+} 2.5 \mathrm{mmol} / \mathrm{L}$ & & & $\mathrm{K}^{+} 3.5 \mathrm{mmol} / \mathrm{L}$ & \\
\hline & $\mathrm{Mg}^{2+} 1 \mathrm{mmol} / \mathrm{L}$ & & & $\mathrm{Ca}^{2+} 2.15 \mathrm{mmol} / \mathrm{L}$ & \\
\hline & $\mathrm{Cl}^{-} 127 \mathrm{mmol} / \mathrm{L}$ & & & $\mathrm{Mg}^{2+} 0.9 \mathrm{mmol} / \mathrm{L}$ & \\
\hline & Acetate 24 mmol/L & & & $\mathrm{Cl}^{-} 109 \mathrm{mmol} / \mathrm{L}$ & \\
\hline & \multirow[t]{2}{*}{ Malate $5 \mathrm{mmol} / \mathrm{L}$} & & & Acetate $20.7 \mathrm{mmol} / \mathrm{L}$ & \\
\hline & & & & Malate $4.3 \mathrm{mmol} / \mathrm{L}$ & \\
\hline \multirow[t]{7}{*}{ Isopedia ${ }^{M D, a, b}$} & Glucose 1\% & \multirow[t]{7}{*}{351} & \multirow[t]{7}{*}{$\begin{array}{l}60 \mathrm{~mL} \text { of glucose } 30 \% \text { per } \\
500 \mathrm{~mL}\end{array}$} & Glucose $4.2 \%$ & \multirow[t]{7}{*}{495} \\
\hline & $\mathrm{Na}^{+} 140 \mathrm{mmol} / \mathrm{L}$ & & & $\mathrm{Na}^{+} 125 \mathrm{mmol} / \mathrm{L}$ & \\
\hline & $\mathrm{K}^{+} 4 \mathrm{mmol} / \mathrm{L}$ & & & $\mathrm{K}^{+} 3.6 \mathrm{mmol} / \mathrm{L}$ & \\
\hline & $\mathrm{Ca}^{2+} 1 \mathrm{mmol} / \mathrm{L}$ & & & $\mathrm{Ca}^{2+} 0.9 \mathrm{mmol} / \mathrm{L}$ & \\
\hline & $\mathrm{Mg}^{2+} 1 \mathrm{mmol} / \mathrm{L}$ & & & $\mathrm{Mg}^{2+} 0.9 \mathrm{mmol} / \mathrm{L}$ & \\
\hline & $\mathrm{Cl}^{-} 118 \mathrm{mmol} / \mathrm{L}$ & & & $\mathrm{Cl}^{-} 107 \mathrm{mmol} / \mathrm{L}$ & \\
\hline & Acetate $30 \mathrm{mmol} / \mathrm{L}$ & & & Acetate $27 \mathrm{mmol} / \mathrm{L}$ & \\
\hline Glucidion G5 & Glucose $5 \%$ & \multirow[t]{4}{*}{460} & \multirow[t]{4}{*}{$\begin{array}{l}10 \mathrm{~mL} \text { of } \mathrm{NaCl}^{-} 2 \mathrm{~g} / 10 \mathrm{~mL} \\
\text { per } 500 \mathrm{~mL}\end{array}$} & Glucose $4.9 \%$ & \multirow[t]{4}{*}{585} \\
\hline OsmotanG5 & $\mathrm{Na}^{+} 68.4 \mathrm{mmol} / \mathrm{L}$ & & & $\mathrm{Na}^{+} 136 \mathrm{mmol} / \mathrm{L}$ & \\
\hline BionolyteG5 $5^{\mathrm{MD}}$ & $\mathrm{K}^{+} 26.8 \mathrm{mmol} / \mathrm{L}$ & & & $\mathrm{K}^{+} 26.2 \mathrm{mmol} / \mathrm{L}$ & \\
\hline Polyionique $\mathrm{G} 5^{\mathrm{MD}}$ & $\mathrm{Cl}^{-} 95.2 \mathrm{mmol} / \mathrm{L}$ & & & $\mathrm{Cl}^{-} 163 \mathrm{mmol} / \mathrm{L}$ & \\
\hline \multirow[t]{5}{*}{ Dextrion G5\% ${ }^{\mathrm{MD}}$} & Glucose 5\% & \multirow[t]{5}{*}{393} & \multirow{5}{*}{$\begin{array}{l}15 \mathrm{~mL} \text { of } \mathrm{NaCl}^{-} 2 \mathrm{~g} / 10 \mathrm{~mL} \\
\quad \text { per } 500 \mathrm{~mL}\end{array}$} & Glucose $4.8 \%$ & \multirow[t]{5}{*}{582} \\
\hline & $\mathrm{Na}^{+} 34.2 \mathrm{mmol} / \mathrm{L}$ & & & $\mathrm{Na}^{+} 136 \mathrm{mmol} / \mathrm{L}$ & \\
\hline & $\mathrm{K}^{+} 20.1 \mathrm{mmol} / \mathrm{L}$ & & & $\mathrm{K}^{+} 19.5 \mathrm{mmol} / \mathrm{L}$ & \\
\hline & $\mathrm{Ca}^{2+} 2.23 \mathrm{mmol} / \mathrm{L}$ & & & $\mathrm{Ca}^{2+} 2.2 \mathrm{mmol} / \mathrm{L}$ & \\
\hline & $\mathrm{Cl}^{-} 54.3 \mathrm{mmol} / \mathrm{L}$ & & & $\mathrm{Cl}^{-} 156 \mathrm{mmol} / \mathrm{L}$ & \\
\hline
\end{tabular}

$\mathrm{Na}$, sodium; $K$, potassium; $\mathrm{Cl}$, chlorine; $\mathrm{Ca}$, calcium; $\mathrm{Mg}$, magnesium

a isotonic fluid

${ }^{\mathrm{b}}$ balanced fluid 
the UK [15], the Institute for Safe Medication Practices of Canada [14], and the USA [16] warned about the dangers of infusing hypotonic intravenous fluids in children. This case outlines once again the importance of updating anchored hazardous clinical practices according to actual recommendations. The American Academy of Pediatrics published a clinical practice guideline in December 2018 strongly recommending that patients aged from 28 days to 18 years receive isotonic solutions when MIVF is needed [10] (Table 1). The strength of this recommendation should overcome the remaining concerns about the safety of isotonic MIVF in acutely ill children. Isotonic saline solutions have shown a protective effect against hyponatremia [17-19] without any significant association with deleterious effects such as fluid overload, hypernatremia, or phlebitis [20]. Besides, the amount of fluid intake should be adapted to every patients' clinical situation, for there is no one-size-fits-all formula [11]. For example, one of the main causes of acquired hyponatremia during hospitalization in pediatrics is SIAD [11]. SIAD is characterized by excessive arginine-vasopressine (AVP) secretion leading to free-water retention that induces a decrease in plasmatic osmolarity associated with intracellular overhydration [9]. SIAD management essentially involves fluid intake restriction $\left(600 \mathrm{~mL} / \mathrm{m}^{2} /\right.$ day) [13] and sufficient sodium intake $(6-10 \mathrm{~g} /$ day $)$ to compensate for relative hypovolemia induced by intracellular fluid shift and associated reduction in plasma water volume [9]. Pediatricians should also realize that fluid overload is the consequence of the total amount of fluid intake more than the fluid's tonicity, as is still commonly thought [11].

The patient's natremia was rapidly corrected when hypertonic saline infusion was started, with an increase of $15 \mathrm{mmol} / \mathrm{L}$ in the first 5 hours $(3 \mathrm{mmol} / \mathrm{L} /$ hour $)$, and an overall increase of $40 \mathrm{mmol} / \mathrm{L}$ in the first 24 hours. Management of acute hyponatremia should be based on symptom severity rather than on sole natremia [13]. Recommended treatment for acute symptomatic hyponatremia ranges from 1 to $3 \mathrm{~mL} / \mathrm{kg}$ ( 0.5 to $1.5 \mathrm{mmol} / \mathrm{kg}$ ) of intravenous $\mathrm{NaCl} 3 \% \mathrm{w} / \mathrm{v}$ administered over 10-15 minutes $[13,21,22]$. Additional boluses are to be administered if the patient's condition does not improve, with a target increase of $5 \mathrm{mmol} / \mathrm{L}$ in sodium concentration in the first 1-2 hours [13] to avoid ODS [21, 22]. ODS remains extremely rare and is more likely to occur in patients with concomitant risk factors as well as in the context of chronic hyponatremia [23]. The correction in the first 48 hours should not exceed $15-20 \mathrm{mmol} / \mathrm{L}$ [13]. In this case, the natremia correction rate was further accelerated by the temporary polyuria that appeared a few hours after initiation of the hypertonic infusion. Desmopressin administration allowed for a small reduction in the sodium correction rate. Despite the persistence of inappropriate AVP secretion, after a few days, there was an increase in diuretic activity with a decrease in urinary osmolarity [9]. This physiological response, which varies according to the individual and the etiology of hyponatremia, intends to maintain homeostasis and counteract the harmful effects of the water retention from SIAD [9].

\section{Conclusion}

This case highlights the importance of using isotonic solutions in children whenever MIVFs are required. It is an occasion to remind pediatricians of the American Academy of Pediatrics' recommendations regarding the use of isotonic MIVF in children.

\section{Abbreviations}

aEEG: Amplitude-integrated electroencephalography; BP: Blood pressure; Hb: Hemoglobin; HR: Heart rate; MIVF: Maintenance intravenous fluids; MRI: Magnetic resonance imaging; ODS: Osmotic demyelination syndrome; PICU: Pediatric Intensive Care Unit; PI: Pulsatility Index; Plt: Platelets; RR: Respiratory rate; SIAD: Syndrome of inappropriate antidiuresis; TDU: Transcranial Doppler ultrasound; Vd: Diastolic velocity; WBC: White blood cells.

\section{Acknowledgements \\ Not applicable.}

\section{Authors' contributions}

$M D, E L$, and ADG performed the data collection, drafted the initial manuscript, and reviewed and revised the manuscript. FP, BL, and NL reviewed and revised the manuscript. IG contributed to the conception of the manuscript and interpretation of the data, critically reviewed and revised the manuscript, and, as English speaker, performed English editing. DB contributed to the conception of the manuscript and the interpretation of the data, coordinated and supervised data collection, drafted the initial manuscript, and reviewed and revised the manuscript. All authors took care of the patient and approved the final manuscript as submitted and agree to be accountable for all aspects of the work. All authors read and approved the final manuscript.

Funding

No funding was secured for this study.

Availability of data and materials

All data supporting the findings of this article are included in the manuscript.

\section{Declarations}

Ethics approval and consent to participate

Not applicable.

\section{Consent for publication}

Written informed consent was obtained from the patient's legal guardians for publication of this case report and any accompanying images. A copy of the written consent is available for review by the Editor-in-Chief of this journal.

\section{Competing interests}

The authors declare that they have no competing interest to disclose.

\section{Author details}

${ }^{1}$ Pediatric Intensive Care Unit, CHU de Caen, 14000 Caen, France. ${ }^{2}$ Pediatric Intensive Care Unit, CHU de Nantes, 44000 Nantes, France. ${ }^{3}$ CNRS, INSERM, I'institut du thorax, Université de Nantes, CHU Nantes, 44000 Nantes, France. 
${ }^{4}$ Pediatric Department, CHU de Rouen, 76000 Rouen, France. ${ }^{5}$ Medical School, Université de Rouen, 76000 Rouen, France. ${ }^{6}$ Pediatric Nephrology, CHU de Nantes, 44000 Nantes, France. ${ }^{7}$ Institut du thorax, INSERM, CNRS, UNIV Nantes, 44000 Nantes, France. ${ }^{8}$ Department of Pharmacy, CHU de Caen, 14000 Caen, France. ${ }^{9}$ Medical School, Université Caen Normandie, 14000 Caen, France.

${ }^{10}$ Service de reanimation pédiatrique, $\mathrm{CHU}$ de Nantes, 4ème étage bâtiment HME, 38 boulevard Jean-Monnet, 44093 Nantes Cedex 1, France.

Received: 16 November 2020 Accepted: 3 May 2021

Published online: 07 June 2021

\section{References}

1. Freeman MA, Ayus JC, Moritz ML. Maintenance intravenous fluid prescribing practices among paediatric residents. Acta Paediatr. 2012;101:e465-468.

2. Holliday MA, Segar WE. The maintenance need for water in parenteral fluid therapy. Pediatrics. 1957;19:823-32.

3. Hoorn EJ, Geary D, Robb M, Halperin ML, Bohn D. Acute hyponatremia related to intravenous fluid administration in hospitalized children: an observational study. Pediatrics. 2004;113:1279-84.

4. Moritz ML, Ayus JC. Prevention of hospital-acquired hyponatremia: a case for using isotonic saline. Pediatrics. 2003;111:227-30.

5. Devaraj NK. Case report: a case of pericardial effusion presenting to primary care. Med J Malays. 2017;72:252-3.

6. Devaraj NK. Antibiotic resistance: a real menace. Oman Med J. 2017;32:531.

7. Teoh SH, Draman N, Devaraj NK. Pelvic actinomycosis: a report of two cases with different presentations. p. 3.

8. Arieff Al, Ayus JC, Fraser CL. Hyponatraemia and death or permanent brain damage in healthy children. BMJ. 1992;304:1218-22.

9. Frouget T. The syndrome of inappropriate antidiuresis. Rev Med Interne. 2012;33:556-66

10. Feld LG, Neuspiel DR, Foster BA, Leu MG, Garber MD, Austin K, et al. Clinical practice guideline: maintenance intravenous fluids in children. Pediatrics. 2018;142:e20183083.

11. Moritz ML, Ayus JC. Maintenance intravenous fluids in acutely ill patients. N Engl J Med. 2015;373:1350-60.
12. Karageorgos SA, Kratimenos P, Landicho A, Haratz J, Argentine L, Jain A, et al. Hospital-acquired hyponatremia in children following hypotonic versus isotonic intravenous fluids infusion. Children (Basel). 2018;5:139.

13. Moritz ML. Syndrome of inappropriate antidiuresis. Pediatr Clin N Am. 2019;66:209-26.

14. Koczmara C, Wade AW, Skippen P, Campigotto MJ, Streitenberger K, Carr $R$, et al. Hospital-acquired acute hyponatremia and reports of pediatric deaths. Dynamics. 2010;21:21-6.

15. 2007-NRLS-0409-Hyponatraemia-cen-PSA-2007-03-28-v1.pdf. https:// www.sps.nhs.uk/wp-content/uploads/2018/02/2007-NRLS-0409-Hypon atraemia-cen-PSA-2007-03-28-v1.pdf. Accessed 23 Feb 2019.

16. Alert IM. Plain D5W or hypotonic saline solutions post-op could result in acute hyponatremia and death in healthy children. Alta RN. 2010;66:16-9.

17. McNab S. Isotonic vs hypotonic intravenous fluids for hospitalized children. JAMA. 2015;314:720-1.

18. Montañana PA, Modesto i Alapont V, Ocón AP, López PO, López Prats $J$, Toledo Parreño JD. The use of isotonic fluid as maintenance therapy prevents iatrogenic hyponatremia in pediatrics: a randomized, controlled open study. Pediatr Crit Care Med. 2008;9:589-97.

19. Foster BA, Tom D, Hill V. Hypotonic versus isotonic fluids in hospitalized children: a systematic review and meta-analysis. J Pediatr. 2014;165:163169.e2.

20. Alves JTL, Troster EJ, de Oliveira CAC. Isotonic saline solution as maintenance intravenous fluid therapy to prevent acquired hyponatremia in hospitalized children. J Pediatr (Rio J). 2011;87:478-86.

21. Spasovski G, Vanholder R, Allolio B, Annane D, Ball S, Bichet D, et al. Clinica practice guideline on diagnosis and treatment of hyponatraemia. Eur J Endocrinol. 2014;170:G1-47.

22. Lavagno C, Milani GP, Uestuener P, Simonetti GD, Casaulta C, Bianchetti $M G$, et al. Hyponatremia in children with acute respiratory infections: a reappraisal. Pediatr Pulmonol. 2017;52:962-7.

23. King JD, Rosner MH. Osmotic demyelination syndrome. Am J Med Sci. 2010;339:561-7.

\section{Publisher's Note}

Springer Nature remains neutral with regard to jurisdictional claims in published maps and institutional affiliations.
Ready to submit your research? Choose BMC and benefit from:

- fast, convenient online submission

- thorough peer review by experienced researchers in your field

- rapid publication on acceptance

- support for research data, including large and complex data types

- gold Open Access which fosters wider collaboration and increased citations

- maximum visibility for your research: over $100 \mathrm{M}$ website views per year

At BMC, research is always in progress.

Learn more biomedcentral.com/submissions 